\title{
Estudio de los vínculos entre cultura organizacional y calidad de los servicios educativos en la imagen institucional de una universidad privada confesional
}

\author{
Josué Turpo y Donald Jaimes ${ }^{1}$ \\ ${ }^{1}$ Universidad Peruana Unión
}

Recibido: 04 de agosto de 2016

Aceptado: 01 de diciembre de 2017

\begin{abstract}
Resumen
La calidad de los servicios académicos, junto con la imagen institucional y la cultura organizacional son factores clave en el proceso de licenciamiento universitario. El objetivo de este artículo es determinar la relación que tiene la calidad de los servicios académicos y la cultura organizacional en la imagen institucional de una institución privada confesional. La metodología utilizada es correlacional bajo el enfoque cuantitativo. Se trata de una correlación múltiple con propósito de causalidad que pretende explicar las relaciones existentes, a través del análisis de regresión lineal múltiple ANOVA. Se aplicaron tres instrumentos, el Organizational Culture Survey (DOCS) de Denison de 60 ítems; el Instrumento de evaluación escala de Satisfacción Estudiantil en cuanto a la Calidad Educativa (SEICE) 93 ítems y el cuestionario de Imagen Institucional para públicos selectos de Escobar de 31 ítems. Los instrumentos de cultura y calidad utilizaron como evaluación la escala tipo Likert de cinco puntos, en el caso del instrumento de imagen se calificó a través signos positivos y negativos. Los instrumentos fueron aplicados a una población de 221 personas del público alumnos de una universidad privada confesional. Entre los resultados se obtuvieron relaciones significativas entre las variables estudiadas y sus dimensiones. Se concluye que la valoración positiva de la imagen institucional se ve afectada por la cultura organizacional y la calidad de los servicios educativos.
\end{abstract}

Palabras clave: cultura institucional, imagen institucional, calidad de los servicios educativos, educación superior, universidad confesional.

\footnotetext{
${ }^{1}$ Correspondencia al autor

E-mail: josuetc@upeu.edu.pe
} 


\begin{abstract}
The quality of academic services, along with the institutional image and organizational culture are key factors in the university licensing process. This study explores the relationship between the quality of academic services and the organizational culture in the institutional image of a private denominational institution. It's a correlational research and used a quantitative approach. It is a multiple purpose causal correlation that seeks to explain existing relationships, through multiple linear regression analysis ANOVA. Three instruments were applied: Denison's 60-item Organizational Culture Survey (DOCS); The Student Satisfaction Scale Instrument for Educational Quality (SEICE) of 93 items and the Escobar's Institutional Image questionnaire for selected audiences of 31 items. The culture and quality instruments used as an evaluation the Likert scale of five points, in the case of the image instrument through positive and negative signs was scored. The instruments were applied to a population of 221 people of the public students of a confessional private university. Among the results, significant relationships were obtained between the studied variables and their dimensions. We conclude that the positive evaluation of the institutional image is affected by the organizational culture and the quality of educational services.
\end{abstract}

Keywords: Institutional Culture, Institutional Image, Quality of Academic Services, Higher Education, Confessional University.

\title{
Introducción
}

El tema de la calidad universitaria está ligado a los procesos de licenciamiento que se vienen desarrollando en los últimos cinco años en el Perú. Las instituciones de educación superior están desempeñándose en alcanzar los indicadores con tal de ser llamados institución acreditada. Por ejemplo, en el tercer ranking de calidad universitaria del Perú, realizado por América Economía Intelligence (2012), se presenta un panorama completo de la oferta de educación del Perú; según el cual mediante preguntas específicas, se mide el prestigio de las distintas universidades, el proceso de decisión de los postulantes, el índice de empleabilidad, así como el índice de investigación, concluyendo que si las universidades peruanas no generan acuerdos para integrarse a estándares comunes de medición de calidad, tampoco quedarán unidos frente al sinnúmero de aspectos que requieren una solución común.. Por lo tanto, resulta muy importante para los procesos de calidad universitaria seleccionar los factores relacionados a este proceso.

Topor (1986) da tres razones fundamentales por las que una escuela debe ocuparse en hacer una investigación sobre su imagen entre sus públicos: (1) Es necesario conocer cómo es percibida 
su institución en relación con instituciones competidoras. (2) Es necesario conocer cómo es percibida por sus públicos. (3) Necesita monitorear y mejorar la imagen percibida en forma constante. Padres, maestros, alumnos y otros públicos pueden tener imágenes distintas de la escuela, basándose en lo que en forma particular han experimentado y oído de la institución (Renihan y Renihan, 1989). Es imposible para una institución no tener una imagen y que los administradores están altamente comprometidos en desarrollar una imagen favorable de su institución ante los ojos de sus públicos.

Perozo de Jiménez (2004) resalta la importancia de realizar estudios sobre imagen corporativa. Hoy existen muchos artículos que hablan de la importancia de desarrollar estudios sobre la imagen corporativa y cómo afecta positivamente el éxito organizacional (Briançon Messinger, 2007; Lojo, 2011).

Ernesto, Varela, \& Irlanda (2011, p. 97) menciona que "la cultura organizacional es un elemento integrador Los estudiosos de la $\mathrm{CO}$, consideran que ésta es el marco de referencia de los integrantes de una organización $\mathrm{y}$, al mismo tiempo, proporciona lineamientos de comportamiento".

Este interés sobre la imagen institucional ha dado lugar a un número creciente de estudios y propuestas, que van desde investigaciones de mercado, estudios sobre cultura organizacional, calidad de servicios educativos y estudios de marca e imagen institucional. Las investigaciones anteriores han sugerido que la imagen institucional está relacionada la cultura organizacional (Meza, 1996, 2011; Miranda, 2010; Solís, 2004; Acevedo, 2008, Ogliastri et al, 1999, Turpo, 2011).

Esta investigación responde a los propósitos: (1) determinar la relación que tiene la calidad de los servicios académicos y la cultura organizacional en la imagen institucional de una institución privada confesional percibida por el público alumnos (2) usar la información recolectada para hacer sugerencias a la administración que faciliten mejoras en los servicios educativos de la universidad.

\section{Concepto sobre cultura organizacional}

Para este estudio es necesario aproximarse al significado o concepto exacto de cultura 
organizacional. Barragán y Caballero (2008) argumentan que la cultura es un factor determinante y explicativo del desempeño organizacional. Pérez (1993) arguye que cada organización puede ser estudiada como poseedora de un sistema cultural específico (conocido como subcultura). Según García (2006) cultura organizacional debe tener una aproximación a través de una metodología cualitativa, a través de observaciones de campo. La autora comenta que en el caso de cultura se deben analizar valores y presunciones subyacentes.

Salazar Estrada, Guerrero Pupo, Machado Rodríguez, \& Cañedo Andalia, (2009, p. 69) mencionan sobre cultura organizacional: "Esta comprende el patrón general de conductas, creencias y valores compartidos por los miembros de una organización. Los miembros de la organización determinan en gran parte su cultura”. Por ello la definición que se utiliza está relacionado a estos componentes.

\section{Aproximaciones al concepto de calidad universitaria}

En la última década, la calidad se ha convertido en un concepto citado por las principales instituciones públicas y de servicios, se ha convertido en una meta buscada de una manera completa, ya que se ha considerado de forma común que lo que tiene "calidad" satisface las expectativas del cliente, la calidad en general abarca todas las cualidades de un producto o un servicio, cuando sus características, tangibles e intangibles satisfacen las necesidades del usuario (Cantú 2001, citado por Gómez, 2002).

Salinas, Morales y Martínez (2008) comentan que lo que hasta hace algunos años fue dominio exclusivo de la universidad y de la educación superior, ahora es un territorio de competencia abierta que obliga a los universitarios a redefinir su papel y el de la educación superior. En efecto, durante la última década, la preocupación por la evaluación de la calidad de la educación universitaria constituye un rasgo esencial de la educación superior en los países más desarrollados.

\section{Imagen institucional y sus aproximaciones conceptuales}

Es de gran valor sustentar en la psicología el concepto de percepción. Smirnov (1960) la define como el reflejo del conjunto de cualidades y partes de los objetos y fenómenos de la realidad, los cuales actúan directamente sobre los órganos de los sentidos. Las percepciones, así como las sensaciones, son el resultado de la acción directa de los objetos sobre los órganos de los sentidos. 
La percepción es siempre una imagen más o menos complicada del objeto.

Lojo (2011, p. 86) manifiesta que "la Imagen Corporativa se forma en la mente de los públicos a partir del procesamiento de la información que recibe sobre la organización". En tal sentido, la imagen es el resultado de la información que recibe un sujeto sobre la organización.

En el contexto de la escuela y el sistema educativo, Renihan y Renihan (1989) definen la imagen como la suma de opiniones subjetivas acerca de la calidad del aprendizaje y el ambiente social. Agregan que es el sentimiento colectivo desarrollado por públicos diversos como resultado de observaciones y experiencias de la escuela, acumuladas a lo largo de los años. Barich y Kotler (1991) usan el término imagen para representar la suma de creencias, actitudes e impresiones que una persona o un grupo tienen de un objeto. El objeto puede ser una compañía, producto, marca, lugar o persona. La impresión puede ser verdadera o falsa, real o imaginaria, correcta o equivocada.

\section{Definiciones de públicos selectos}

Con relación a los públicos, Simon (1986) declara, que no hay uno sino muchos públicos. Todos pertenecemos al mismo tiempo a un número casi infinito de públicos. Se puede ser protestante, miembro de un sindicato, afiliado a algún club, un votante, un joven, un deportista, etc.

Yasky (2011, p. 66) menciona:

Es importante identificar a las personas o instituciones que se involucran en la actividad. De hecho, los públicos son quienes están afectados e implicados en la vida empresaria cotidiana. Son los actores destacados en este ámbito, son informantes claves que participan activamente...

Marston (1981) clasifica en públicos internos y externos. El público interno está constituido por personas conectadas a una organización y con quienes la organización se comunica normalmente en la rutina ordinaria de trabajo. Entre los públicos internos, se encuentran: 1) empleados; 2) accionistas; 3) proveedores; 4) clientes y 5) vecinos de la empresa. También identifica a públicos internos de las escuelas: 1) empleados; 2) estudiantes; 3) padres; 4) proveedores; 5) público en general.

Kotler (1988) define como meta principal de una empresa la satisfacción de las necesidades específicas de su mercado principal. Para lograrlo debe mantener una estrecha relación con sus 
proveedores e intermediarios.

Según Hartman y Schmidt (1995), en los recientes años, se ha incrementado el interés de las instituciones de educación superior en grupos de legisladores, medios de comunicación, padres y el público en general, quienes demandan mayor responsabilidad de los colegios y universidades.

Kotler y Fox (1985) definen el concepto de público como grupos distintos de personas y organizaciones que tienen un actual y potencial interés y que afectan una institución. Identifican dieciséis grupos de públicos de una institución educativa (Figura 3).

La determinación de los públicos más importantes de una universidad, es una actividad clave para determinar y describir las necesidades específicas de los mismos. Topor (1983) especifica que esta segmentación le ayudará a predecir conductas y a establecer programas de atención a las necesidades detectadas. Los públicos son segmentados en forma demográfica (edad, sexo, educación, etc.); geográfica (región, clima, densidad de población, etc.) y psicográfica (personalidad, estilo de vida, factores de riesgo, etc.).

Este estudio pretende plantear una investigación acerca de la percepción que tienen los estudiantes sobre la imagen institucional en relación a la cultura organizacional y calidad de los servicios educativos en una universidad privada confesional.

\section{Método}

\section{Participantes}

Se reclutó a: 221 estudiantes de ambos sexos, alumnos de cinco carreras universitarias de la Universidad Privada Confesional.

\section{Materiales y procedimiento}

El tipo de investigación es descriptivo, correlacional, transaccional, en coherencia con el objetivo de establecer relaciones entre las variables cultura organizacional, imagen institucional y calidad de los servicios según la percepción de los alumnos, padres y público externo (Acuña, 2011; Hernández, Fernández y Baptista, 2003). Esta investigación se encuentra ubicada en el estudio cuantitativo, pues para la medición de las variables se cuenta con tres cuestionarios tipo Likert. El 
método de recolección de datos fue la encuesta y los Instrumento utilizados fueron el Cuestionario sobre cultura organizacional de Denison: [Versión] Denison Organizational Culture Survey (DOCS). Instrumento de evaluación escala de Satisfacción Estudiantil en cuanto a la Calidad Educativa (SEICE) desarrollado por Bullón (2007). Cuestionario de imagen institucional para públicos selectos preparado por la Meza Escobar $(1999,2011)$ en donde fue incluida información sobre datos demográficos tales como el género y edad. Los instrumentos tomaron en cuenta las implicaciones teóricas sobre las variables elegidas y sobre la base de búsqueda bibliográfica extensa, incluyendo instrumentos previos sobre el tema.

\section{Instrumentos utilizados y validación}

El instrumento de imagen institucional constó de dos secciones: Primera sección. Compuesta por variables sociodemográficas. Se solicitaron datos personales a cada uno de los públicos. Segunda sección. Está integrada por treinta ítems, los cuales son adjetivos calificativos bipolares, mediante los cuales se determinó un perfil de la Universidad Privada Confesional. Generalmente del lado izquierdo se encuentran adjetivos de evaluación-positivos, de potenciafortalezas y de actividad. Del lado derecho se encuentran los adjetivos extremos de evaluaciónnegativos, de potencia-debilidades y de pasividad. Se utilizó como adjetivos extremos palabras antónimas. En algunos ítems no son necesariamente antónimos, sino adjetivos que pueden ser comprendidos con mayor facilidad por los participantes. Cada uno de los treinta ítems se agrupan, tentativamente, en las siguientes dimensiones: (1) espiritual (1-7); (2) intelectual (8-16); (3) social (17-24); (4) física (25-31).

La calificación se realizó de la siguiente forma. Una sección, en el medio, la componen siete columnas correspondientes a la escala ordinal utilizada para evaluar cada ítem. La columna central (4a) está destinada para respuestas de empate o no sé. Se dividió con una diagonal en cada renglón. Las tres columnas de la izquierda corresponden a la medida intenso (1a), moderado (2a) y regular (3a), con respecto a adjetivos positivos, fortalezas y actividades. Las tres columnas de la derecha corresponden a la medida de regular (5a), moderado (6a) e intenso (7a), con respecto a adjetivos negativos, debilidades y pasividad. Dentro de la calificación se consideraron signos 
positivos y negativos. El positivo indica hábito o actitud adecuada, y el negativo indica hábito o actitud inadecuada. Además existen puntajes que se denominan ponderados, tienen la finalidad de indicar cuál es el nivel de proporción en el que se presenta el hábito o la actitud en determinado grupo de sujetos, sea de buen rendimiento o de bajo rendimiento. Por ejemplo, entre un puntaje 7, con signo positivo, y otro, 13 con signo positivo, el segundo, por ser mayor, es el mejor. El puntaje 0 dentro de la calificación, significa que no es hábito o actitud inadecuada, ni tampoco adecuada porque pertenece en igual proporción, tanto a los sujetos de bajo rendimiento como a los de buen rendimiento.

El instrumento de cultura organizacional está integrado por 60 ítems que se agrupan, tentativamente, en las siguientes dimensiones: (1) cultura social (1-15); (2) cultura espiritual (1630); (3) cultura física (31-454); (4) cultura intelectual (46-60).

El instrumento de calidad de servicios educativos es integrado por 93 ítems que se agrupan, tentativamente, en las siguientes dimensiones: (1) calidad de los servicios intelectuales (1-34); (2) calidad de los servicios físicos (35,50; 76-93); (3) calidad de los servicios sociales (51-58; 67-75); (4) calidad de los servicios espirituales (59-66).

En el caso de la encuesta de cultura organizacional y calidad de servicios educativos, su sistema de calificación se basó en la escala de Likert.

En la prueba piloto también verificamos la confiabilidad, comparando los resultados obtenidos, también para aplicar la validez de criterio. En la cultura organizacional, el coeficiente Alfa de Cronbach fue de 0.929 lo cual permite decir que el instrumento empleado en la prueba piloto para medir la cultura organizacional tiene un alto nivel de confiabilidad. A la vez se determinó la validez estadística de cada ítem por el mismo procedimiento de Alfa de Cronbach aplicando el comando "si se elimina el elemento". En este cuadro de validez ítem por ítem, en relación con el nivel de coeficiente alfa, se observa que generalmente los ítems generan una correlación positiva alta. Esto indica que los ítems están midiendo lo mismo que el instrumento. Por lo cual, no requiere que se elimine ningún elemento.

En el caso de la confiabilidad de la calidad de los servicios educativos, el coeficiente Alfa de Cronbach es de 0.979 (tabla 02), lo cual permite decir que el instrumento empleado en la prueba piloto para medir la calidad de servicios educativos tiene un nivel alto de confiabilidad. 
A la misma vez se determinó la validez estadística de cada ítem por el mismo procedimiento de Alfa de Cronbach aplicando el comando "si se elimina el elemento". En este cuadro de validez ítem por ítem, en relación con el nivel de coeficiente alfa, se observa que generalmente los ítems generan una correlación alta. Esto indica que los ítems están midiendo lo mismo que el instrumento. Por lo cual, no requiere que se elimine ningún elemento.

En el caso de imagen institucional, el coeficiente Alfa de Cronbach es de 0.930 (tabla 03), lo cual permite decir que el instrumento empleado en la prueba piloto para medir la imagen institucional tiene un nivel alto de confiabilidad. A la misma vez se determinó la validez estadística de cada ítem por el mismo procedimiento de Alfa de Cronbach, aplicando el comando "si se elimina el elemento". En este cuadro de validez ítem por ítem en relación con el nivel de coeficiente alfa, se observa que generalmente los ítems generan una correlación alta. Esto indica que están midiendo lo mismo que el instrumento. Por lo que no se requiere eliminar ningún elemento.

\section{Recolección de datos}

Para la recolección de datos se realizó los siguientes pasos: En cuanto a los alumnos. Se solicitó a la Administración de la Universidad Privada Confesional ubicada en la ciudad de Lima, Perú, la autorización para aplicar los cuestionarios de Imagen institucional, Cultura organizacional y Calidad de servicios educativos. Se usó el análisis de estadística descriptiva y la estadística no paramétrica. Análisis de regresión lineal múltiple ANOVA a través de 07 pasos. El paquete fue el estadístico SPSS 20.0 en español con el procedimiento de eliminación de variables.

\section{Resultados}

Los análisis se enfocan en las correlaciones entre dimensiones cultura organizacional e imagen institucional en el público alumnos. En la tabla comprobamos la correlación de las variables utilizando la hipótesis nula H_0: $\rho \leq 0$ Se dan los coeficientes de correlación de Pearson muestrales y la significación de contraste. 
Tabla 01

Correlación Cultura organizacional e imagen institucional

\begin{tabular}{|c|c|c|c|c|c|c|}
\hline & & $\begin{array}{c}\text { Imagen } \\
\text { Institucional }\end{array}$ & $\begin{array}{l}\text { Cultura } \\
\text { social }\end{array}$ & $\begin{array}{l}\text { Cultura } \\
\text { espiritual }\end{array}$ & $\begin{array}{l}\text { Cultura } \\
\text { física }\end{array}$ & $\begin{array}{c}\text { Cultura } \\
\text { intelectual }\end{array}$ \\
\hline Correlación & Imagen Institucional & 1,000 &,- 435 &,- 424 &,- 399 &,- 395 \\
\hline \multirow[t]{4}{*}{ de Pearson } & Cultura social &,- 435 & 1,000 & ,678 & ,627 & ,610 \\
\hline & Cultura espiritual &,- 424 & ,678 & 1,000 & ,672 & ,647 \\
\hline & Cultura física &,- 399 & ,627 & ,672 & 1,000 & ,696 \\
\hline & Cultura intelectual &,- 395 & ,610 &, 647 & ,696 & 1,000 \\
\hline Sig. & Imagen Institucional & . &, 000 &, 000 &, 000 & ,000 \\
\hline \multirow[t]{4}{*}{ (unilateral) } & Cultura social &, 000 & &, 000 &, 000 &, 000 \\
\hline & Cultura espiritual &, 000 & ,000 & . &, 000 &, 000 \\
\hline & Cultura física &, 000 & ,000 &, 000 & . &, 000 \\
\hline & Cultura intelectual &, 000 & ,000 &, 000 &, 000 & . \\
\hline \multirow[t]{5}{*}{$\mathrm{N}$} & Imagen Institucional & 221 & 221 & 221 & 221 & 221 \\
\hline & Cultura social & 221 & 221 & 221 & 221 & 221 \\
\hline & Cultura espiritual & 221 & 221 & 221 & 221 & 221 \\
\hline & Cultura física & 221 & 221 & 221 & 221 & 221 \\
\hline & Cultura intelectual & 221 & 221 & 221 & 221 & 221 \\
\hline
\end{tabular}

En la tabla 01 están correladas negativamente todas las variables predictoras con la variable criterio, pues sus p-valores son 0,000, para un nivel del 5\%. Además, los coeficientes de correlación de cultura social, cultura espiritual, cultura física y cultura intelectual están en el intervalo medio $[-0,33 ;-0,66]:-0,435,-0,424$ y -0, 399, -0, 395 respectivamente. 


\section{Tabla 02}

Coeficientes de regresión dimensión cultura organizacional e imagen institucional

\begin{tabular}{|c|c|c|c|c|c|c|c|c|}
\hline \multicolumn{2}{|c|}{ Modelo } & \multicolumn{2}{|c|}{$\begin{array}{l}\text { Coeficientes no } \\
\text { estandarizados }\end{array}$} & \multirow{2}{*}{$\begin{array}{c}\begin{array}{c}\text { Coeficientes } \\
\text { tipificados }\end{array} \\
\text { Beta }\end{array}$} & \multirow[b]{2}{*}{$\mathrm{t}$} & \multirow[b]{2}{*}{ Sig. } & \multicolumn{2}{|c|}{$\begin{array}{c}\text { Intervalo de confianza de } \\
95.0 \% \text { para B }\end{array}$} \\
\hline & & B & Error típ. & & & & $\begin{array}{l}\text { Límite } \\
\text { inferior }\end{array}$ & $\begin{array}{l}\text { Límite } \\
\text { superior }\end{array}$ \\
\hline 1 & (Constante) & 4,486 & 252 & & 17,816 &, 000 & 3,991 & 4,982 \\
\hline & Cultura social alumnos &,- 577 & 071 &,- 435 & $-8,101$ &, 000 &,- 717 &,- 437 \\
\hline 2 & (Constante) & 5,012 & ,293 & & 17,104 &, 000 & 4,436 & 5,589 \\
\hline & Cultura social alumnos &,- 361 & ,095 &,- 272 & $-3,790$ &, 000 &,- 548 &,- 173 \\
\hline & Cultura espiritual alumnos &,- 355 &, 106 &,- 240 & $-3,348$ &, 001 &,- 564 &,- 146 \\
\hline
\end{tabular}

*Variable criterio: Imagen Institucional

El modelo de regresión múltiple para este modelo viene dado por la siguiente ecuación $Y=\alpha_{0}+\alpha_{1} X_{1}+\alpha_{2} X_{2}+\mathrm{e}$, donde $\mathrm{Y}$ es la variable criterio, $X_{1}$ y $X_{2}$ las variables predictoras que entran a formar parte en el modelo y e es el error, la diferencia entre los valores reales y los valores predichos por la regresión. El modelo queda como Imagen institucional = 5,012 - 0, 361 Cultura social $-0.355 \cdot$ Cultura espiritual.

Tabla 03

Resumen de modelo

\begin{tabular}{|c|c|c|c|c|}
\hline Modelo & $\mathrm{R}$ & $\mathrm{R}$ cuadrado & $\mathrm{R}$ cuadrado corregida & Error típ. de la estimación \\
\hline 1 &, $435^{\mathrm{a}}$ &, 189 &, 186 & 693 \\
\hline 2 & $469^{\mathrm{b}}$ & 220 & 214 & 680 \\
\hline
\end{tabular}

a. Variables predictoras: (Constante), Cultura social alumnos

b. Variables predictoras: (Constante), Cultura social alumnos, Cultura espiritual alumnos

c. Variable criterio: Imagen Institucional 
Si consideramos las variables que han sido introducidas en el modelo y el tamaño de la muestra, considerando la $\mathrm{R}$ cuadrado corregida, podemos decir que el modelo final dado por Imagen institucional $=5,012-0,361 \cdot$ Cultura social $-0.355 \cdot$ Cultura espiritual explica un $43.5 \%$ de la variabilidad de Imagen institucional, en el segundo escalon $\mathrm{R}$ ha aumentado a un valor de $46.9 \%$.

El modelo de regresión que hemos dado por la ecuación del plano Imagen institucional = 5,012 - 0, $361 \cdot$ Cultura social - 0.355 Cultura espiritual. Cultura espiritual produce un ajuste moderado en la predicción de la imagen institucional de un estudiante mediante las dimensiones de la cultura organizacional: social y espiritual.

\section{Tabla 04}

Correlación Cultura e imagen institucional

\begin{tabular}{|c|c|c|c|c|c|c|}
\hline & & $\begin{array}{c}\text { Imagen } \\
\text { Institucional }\end{array}$ & $\begin{array}{c}\text { Calidad de } \\
\text { servicios } \\
\text { intelectuales }\end{array}$ & $\begin{array}{l}\text { Calidad de } \\
\text { servicios } \\
\text { físicos }\end{array}$ & $\begin{array}{l}\text { Calidad de } \\
\text { servicios } \\
\text { sociales }\end{array}$ & $\begin{array}{l}\text { Calidad de } \\
\text { servicios } \\
\text { espirituales }\end{array}$ \\
\hline \multirow{5}{*}{$\begin{array}{l}\text { Correlación } \\
\text { de Pearson }\end{array}$} & Imagen Institucional & 1,000 &,- 421 &,- 521 &,- 494 &,- 415 \\
\hline & Cal. Servicios intelectuales &,- 421 & 1,000 & ,696 & 657 & ,705 \\
\hline & Cal. Servicios físicos &,- 521 & 696 & 1,000 & ,772 & 666 \\
\hline & Cal. Servicios sociales &,- 494 & 657 & ,772 & 1,000 & 689 \\
\hline & Cal. Servicios espirituales &,- 415 &, 705 & ,666 & 689 & 1,000 \\
\hline \multirow{5}{*}{$\begin{array}{c}\text { Sig. } \\
\text { (unilateral) }\end{array}$} & Imagen Institucional & . &, 000 &, 000 &, 000 &, 000 \\
\hline & Cal. Servicios intelectuales &, 000 & . & ,000 &, 000 &, 000 \\
\hline & Cal. Servicios físicos & 000 &, 000 & . &, 000 &, 000 \\
\hline & Cal. servicios sociales &, 000 &, 000 &, 000 & . &, 000 \\
\hline & Cal. servicios espirituales &, 000 &, 000 &, 000 &, 000 & . \\
\hline \multirow[t]{5}{*}{$\mathrm{N}$} & Imagen Institucional & 221 & 221 & 221 & 221 & 221 \\
\hline & Cal. Servicios intelectuales & 221 & 221 & 221 & 221 & 221 \\
\hline & Cal. Servicios físicos & 221 & 221 & 221 & 221 & 221 \\
\hline & Cal. Servicios sociales & 221 & 221 & 221 & 221 & 221 \\
\hline & Cal. Servicios espirituales & 221 & 221 & 221 & 221 & 221 \\
\hline
\end{tabular}


En la tabla 04 están correladas negativamente todas las variables predictoras con la variable criterio, pues sus p-valores son 0,000 , para un nivel del 5\%. Además, los coeficientes de correlación de Calidad de servicios intelectuales, calidad de servicios físicos, calidad de servicios sociales y calidad de servicios espirituales se encuentran en el intervalo [-0.33,-0.66] lo que significan u están por encima que existe una correlación negativa moderada. La variable Calidad de servicios espirituales su coeficiente de correlación es la más baja con un valor de 0,415. Esta variable está correladas significativamente con las subvariables Calidad de servicios intelectuales, Calidad de servicios físicos y Calidad de servicios sociales, cuyos valores son: .0,$421 ;-0,521 ;-0,494$ respectivamente.

Tabla 05

Coeficientes de regresión dimensión calidad de servicios educativos e imagen institucional alumnos

\begin{tabular}{|c|c|c|c|c|c|c|c|c|}
\hline \multicolumn{2}{|c|}{ Modelo } & \multicolumn{2}{|c|}{$\begin{array}{l}\text { Coeficientes no } \\
\text { estandarizados }\end{array}$} & \multirow{2}{*}{$\begin{array}{c}\begin{array}{c}\text { Coeficientes } \\
\text { tipificados }\end{array} \\
\text { Beta }\end{array}$} & \multirow[b]{2}{*}{$\mathrm{t}$} & \multirow[b]{2}{*}{ Sig. } & \multicolumn{2}{|c|}{$\begin{array}{c}\text { Intervalo de confianza de } \\
95.0 \% \text { para B }\end{array}$} \\
\hline & & $\mathrm{B}$ & Error típ. & & & & $\begin{array}{l}\text { Límite } \\
\text { inferior }\end{array}$ & $\begin{array}{l}\text { Límite } \\
\text { superior }\end{array}$ \\
\hline \multirow[t]{2}{*}{1} & (Constante) & 4,073 & ,185 & & 22,042 & ,000 & 3,709 & 4,437 \\
\hline & Cal. servicios físicos &,- 595 & ,066 &,- 521 & $-9,014$ &, 000 &,- 725 &,- 465 \\
\hline \multirow[t]{3}{*}{2} & (Constante) & 4,363 & ,216 & & 20,225 &, 000 & 3,938 & 4,789 \\
\hline & Cal. servicios físicos &,- 395 &, 103 &,- 346 & $-3,851$ &, 000 &,- 598 &,- 193 \\
\hline & Cal. servicios sociales &,- 289 &, 114 &,- 227 & $-2,525$ &, 012 &,- 515 &,- 063 \\
\hline
\end{tabular}

a. Variable criterio: Imagen Institucional

El modelo de regresión múltiple para este modelo viene dado por la siguiente ecuación $Y=\alpha_{0}+\alpha_{1} Z_{2}+\alpha_{2} Z_{3}+\mathrm{e}$, donde $\mathrm{Y}$ es la variable criterio, $Z_{2}$ y $Z_{3}$ las variables predictoras que entran a formar parte en el modelo y e es el error, la diferencia entre los valores reales y los valores predichos por la regresión. El modelo queda como Imagen institucional = 4,363 - 0, 395 ·Calidad de servicios físicos $-0.289 \cdot$ Calidad de servicios sociales. 
Tabla 06

Resumen de modelo calidad de servicios educativos e imagen institucional alumnos

\begin{tabular}{|c|c|c|c|c|}
\hline Modelo & $\mathrm{R}$ & $\mathrm{R}$ cuadrado & $\begin{array}{l}\text { R cuadrado } \\
\text { corregida }\end{array}$ & $\begin{array}{l}\text { Error típ. de la } \\
\text { estimación }\end{array}$ \\
\hline 1 &, $521^{\mathrm{a}}$ & ,272 & ,268 & ,671 \\
\hline 2 &, $541^{\mathrm{b}}$ & ,292 & ,286 & ,662 \\
\hline
\end{tabular}

a. Variables predictoras: (Constante), Calidad de servicios físicos

b. Variables predictoras: (Constante), Calidad de servicios físicos, Calidad de servicios sociales

c. Variable criterio: Imagen Institucional

Si consideramos las variables que han sido introducidas en el modelo y el tamaño de la muestra, considerando la $\mathrm{R}$ cuadrado corregida, podemos decir que el modelo final dado por Imagen institucional $=4,363-0,395 \cdot$ Calidad de servicios físicos $-0.289 \cdot$ Calidad de servicios sociales explica un 52.1\% de la variabilidad de Imagen institucional, en el segundo escalón R ha aumentado a un valor de $54.1 \%$.

El modelo de regresión que hemos dado por la ecuación del plano Imagen institucional = 4,363 - 0,395 Calidad de servicios físicos $-0.289 \cdot$ Calidad de servicios sociales $\cdot$ Calidad de servicios educativos produce un ajuste moderado en la predicción de la imagen institucional del público estudiante mediante las dimensiones de la Calidad de servicios físicos y Calidad de servicios sociales. 


\section{Tabla 07}

Correlaciones cultura organizacional, calidad de servicios educativos e imagen institucional alumnos

\begin{tabular}{|c|c|c|c|c|c|c|}
\hline & & $\begin{array}{c}\text { Imagen } \\
\text { Institucional }\end{array}$ & $\begin{array}{c}\text { Cultura } \\
\text { social }\end{array}$ & $\begin{array}{l}\text { Cultura } \\
\text { espiritual }\end{array}$ & $\begin{array}{c}\text { Calidad } \\
\text { servicios } \\
\text { espirituales }\end{array}$ & $\begin{array}{c}\text { Calidad } \\
\text { servicios } \\
\text { sociales }\end{array}$ \\
\hline \multirow{5}{*}{$\begin{array}{l}\text { Correlación } \\
\text { de Pearson }\end{array}$} & Imagen Institucional & 1,000 &,- 451 &,- 440 &,- 413 &,- 494 \\
\hline & Cultura social &,- 451 & 1,000 & ,727 & ,430 &, 533 \\
\hline & Cultura espiritual &,- 440 & ,727 & 1,000 & ,419 &, 522 \\
\hline & Cal. servicios espirituales &,- 413 & ,430 & ,419 & 1,000 & ,689 \\
\hline & Cal. servicios sociales &,- 494 &, 533 &, 522 & 689 & 1,000 \\
\hline \multirow{5}{*}{$\begin{array}{l}\text { Sig. } \\
\text { (unilateral) }\end{array}$} & Imagen Institucional & - & ,000 & ,000 &, 000 &, 000 \\
\hline & Cultura social &, 000 & . &, 000 &, 000 &, 000 \\
\hline & Cultura espiritual &, 000 &, 000 & . &, 000 &, 000 \\
\hline & Cal. servicios espirituales &, 000 &, 000 &, 000 & . &, 000 \\
\hline & Calidad de servicios sociales &, 000 &, 000 & ,000 & ,000 & \\
\hline \multirow[t]{5}{*}{$\bar{N}$} & Imagen Institucional & 221 & 221 & 221 & 221 & 221 \\
\hline & Cultura social & 221 & 221 & 221 & 221 & 221 \\
\hline & Cultura espiritual & 221 & 221 & 221 & 221 & 221 \\
\hline & Cal. servicios espirituales & 221 & 221 & 221 & 221 & 221 \\
\hline & Calidad de servicios sociales & 221 & 221 & 221 & 221 & 221 \\
\hline
\end{tabular}

Están correladas negativamente todas las variables predictoras con la variable criterio, pues sus p-valores son 0,000, para un nivel del 5\%. Además, los coeficientes de correlación de Cultura Social, Cultura espiritual, Calidad de Servicios espirituales y Calidad de servicios sociales; están en el intervalo medio $[-0,33 ;-0,66]$ cuyos valores son: - $0,451,-0,440$ y -0, 413, 0,494 respectivamente. 


\section{Tabla 08}

Coeficientes de regresión

\begin{tabular}{|c|c|c|c|c|c|c|c|c|}
\hline \multirow{2}{*}{\multicolumn{2}{|c|}{ Modelo }} & \multicolumn{2}{|c|}{$\begin{array}{l}\text { Coeficientes no } \\
\text { estandarizados }\end{array}$} & \multirow{2}{*}{$\begin{array}{l}\text { Joeficientes } \\
\text { tipificados } \\
\text { Beta }\end{array}$} & \multirow[b]{2}{*}{$\mathrm{t}$} & \multirow[b]{2}{*}{ Sig. } & \multicolumn{2}{|c|}{$\begin{array}{c}\text { Intervalo de } \\
\text { confianza de } 95.0 \% \\
\text { para B }\end{array}$} \\
\hline & & $B$ & Error típ. & & & & $\begin{array}{l}\text { Límite } \\
\text { inferior }\end{array}$ & $\begin{array}{l}\text { Límite } \\
\text { superior }\end{array}$ \\
\hline \multirow[t]{2}{*}{1} & (Constante) & 4,272 & ,221 & & 19,348 & ,000 & 3,837 & 4,707 \\
\hline & Cal. servicios sociales &,- 630 & 075 &,- 494 & $-8,408$ &, 000 &,- 777 &,- 482 \\
\hline \multirow[t]{3}{*}{2} & (Constante) & 4,968 & ,279 & & 17,814 &, 000 & 4,419 & 5,518 \\
\hline & Cal. servicios sociales &,- 452 & ,086 &,- 354 & $-5,265$ &, 000 &,- 621 &,- 283 \\
\hline & Cultura social &,- 348 & ,089 &,- 262 & $-3,895$ &, 000 &,- 524 &,- 172 \\
\hline
\end{tabular}

a. Variable dependiente: Imagen Institucional

El modelo de regresión múltiple para este modelo viene dado por la siguiente ecuación $Y=$ $\alpha_{0}+\alpha_{1} Z_{3}+\alpha_{2} X_{1}+$ e, donde $Y$ es la variable criterio, $Z_{3}$ y $X_{1}$ las variables predictoras que entran a formar parte en el modelo y e es el error, la diferencia entre los valores reales y los valores predichos por la regresión. El modelo queda como Imagen institucional = 4,968 - 0, $452 \cdot$ Calidad de servicios sociales $-0.348 \cdot$ Cultura social. 
Tabla 09

Resumen del modelo cultura organizacional, calidad de servicios educativos e imagen institucional alumnos

\begin{tabular}{ccccc} 
Modelo & $\mathrm{R}$ & $\mathrm{R}$ cuadrado & $\begin{array}{c}\text { R cuadrado } \\
\text { corregida }\end{array}$ & $\begin{array}{c}\text { Error típ. de la } \\
\text { estimación }\end{array}$ \\
\hline 1 &, $494^{\mathrm{a}}$ &, 244 &, 241 &, 682 \\
2 &, $541^{\mathrm{b}}$ &, 293 &, 287 &, 661
\end{tabular}

a. Variables predictoras: (Constante), Calidad de servicios sociales

b. Variables predictoras: (Constante), Calidad de servicios sociales,

Cultura social alumnos

c. Variable criterio: Imagen Institucional

Si consideramos las variables que han sido introducidas en el modelo y el tamaño de la muestra, considerando la $\mathrm{R}$ cuadrado corregida, podemos decir que el modelo final dado por Imagen institucional $=5,012-0,452 \cdot$ Cultura social $-0.348 \cdot$ Cultura espiritual explica un $49.4 \%$ de la variabilidad de Imagen institucional, en el segundo escalon $\mathrm{R}$ ha aumentado a un valor de $54.1 \%$.

El modelo de regresión que hemos dado por la ecuación del plano Imagen institucional = 4,968 - 0,452 Calidad de servicios sociales $-0.348 \cdot$ Cultura social produce un ajuste moderado en la predicción de la imagen institucional de un estudiante mediante las dimensiones de la Calidad de servicios sociales y cultura social.

\section{Discusión}

La variable Imagen institucional está correlada significativamente con cultura social y cultura espiritual. Asimismo, Imagen institucional esta correlada significativamente con calidad de servicios físicos y calidad de servicios sociales. En el tercer modelo, imagen institucional esta correlada significativamente con calidad de servicios sociales y cultura social. El resultado del estudio indica que la cultura organizacional y la calidad de los servicios educativos de los públicos encuestados influyen en la imagen institucional de la Universidad privada confesional. Los modelos resultantes: con p-valor de $0<\alpha(\alpha=0.05$ o $\alpha=0.01)$ del público estudiantes: Imagen 
institucional $=5,012-0,361 \cdot$ Cultura social $-0.355 \cdot$ Cultura espiritual explica un 46.9\%; Imagen institucional $=4,363-0,395 \cdot$ Calidad de servicios físicos $-0.289 \cdot$ Calidad de servicios sociales explica un 54.1\%; Imagen institucional $=4,968$ - 0, 452 Calidad de servicios sociales $0.348 \cdot$ Cultura social explica un $54,1 \%$.

Esto indica que estas variables influyen positivamente, si su valor aumenta también lo hace el de Imagen institucional. Mediante un sistema de gestión de la cultura organizacional y una orientación hacia la calidad de los servicios, la organización puede mejorar su imagen institucional e incrementar su volumen de alumnos, esto es consistente por lo reportado por Tous y Bermúdez (2011). Estos resultados también están en línea por lo reportado por Saldaña (2013), Bullón (2007), Corimayhua (2011) y Almeida (2012).

Los resultados en los modelos de regresión lineal permiten predecir la imagen institucional en el público estudiantes a partir de la dimensión de calidad de servicios educativos: calidad de servicios sociales y de cultura organizacional en su dimensión cultura social, aunque de forma moderada en un $46,9 \%$ y $54,1 \%$ respectivamente mostrando la existencia de otros predictores.

Esto está en congruencia con el estudio de Meza (2011), quien halló que se encuentran otras variables a ser analizadas: marketing educativo, apoyo de los padres, entre otros. De todas formas se necesitan estudios para evaluar diferentes variables que podrían ser más significativas.

El coeficiente de correlación Pearson en alumnos es .559; lo que indica una correlación moderada y positiva entre la cultura organizacional y la calidad de los servicios educativos. Asimismo el coeficiente de determinación r (cuadrado) muestra que se puede predecir la imagen institucional a partir de la cultura organizacional y la calidad de los servicios educativos.

De modo general, existe una valoración positiva de la imagen institucional en relación con la cultura organizacional y la calidad de los servicios educativos percibidos por los estudiantes, padres, docentes y público externo de la universidad privada confesional. 


\section{Referencias}

Acevedo, G. (2008). Análisis de la cultura organizacional para generar cambios en una institución educativa. Bogotá: Universidad de la Sabana.

América Economía Intelligence. (2012). Ranking - Las Mejores Universidades de Perú - 2012. Recuperado de: http://rankings.americaeconomia.com/2012/las-mejores-universidades-deperu/ranking.php. Visualizado el 25 de noviembre de 2012.

Barich, H., Kotler, P. (1991). A framework for marketing Image management. Sloan Management Review. Winter.

Barragán, F., Caballero, D. (2008). Estudio de la cultura organizacional, desempeño e innovación en Aero República. Tesis no publicada. Bogotá, D.C.: Universidad de la Sabana, Facultad de Ciencias Económicas y Administrativas.

Briançon Messinger, M. (2007). La importancia de la gestión profesional de la imagen corporativa. Punto Cero, 12(14), 59-64. Retrieved from http://www.scielo.org.bo/scielo.php?script=sci_arttext\&pid=S181502762007000100007\&lng=es\&nrm=iso\&tlng=es

Bullon, S. (2007). La satisfacción estudiantil con la calidad educativa de la universidad. Tesis de licenciatura no publicada. Lima: Pontificia Universidad católica del Perú.

Ernesto, O., Varela, T., \& Irlanda, J. L. (2011). Influencia de la cultura organizacional en el desempeño laboral y la productividad de los trabajadores administrativos en instituciones de educación superior. Omnia Año, 17(1), 96-110.

García, I. (2006). La formación del clima psicológico y su relación con los estilos de liderazgo. Tesis de doctorado no publicado. Granada: Universidad de Granada.

Gómez, F. (2002). Calidad educativa y mejora continua. Universidad Autónoma de Baja California, Facultad de Ciencias Químicas e Ingeniería.

Hartman, D., Schmidt, S. (1995). Understanding student satisfaction from a consumer's perspective: The effects of institutional performance and program outcomes. Research in Higher Education. Vol. 36(2). USA: Human Sciences Press. Inc

Kotler, P. (1988). Marketing management. USA: Prentice-Hall, Inc

Kotler, P., Fox, K. (1985). Strategic marketing for educational Institutions. New Yersey: prentice-Hill.

Lojo, A. P. (2011). Los públicos internos en la construcción de la imagen corporativa. Cuadernos Del Centro de Estudios En Diseño Y Comunicación. Ensayos, (35), 85-94. Retrieved from http://www.scielo.org.ar/scielo.php?script=sci_arttext\&pid=S1853$35232011000100008 \& \operatorname{lng}=\mathrm{es} \& n \mathrm{~mm}=\mathrm{iso} \& \mathrm{t} \operatorname{lng}=\mathrm{es}$

Marston, J. (1981). Relaciones Públicas modernas. México: McGraw-Hill 
Meza Escobar, M. (1996). Imagen institucional de la Universidad de Montemorelos percibida por Públicos Selectos. México: Tesis de doctorado no publicada. Universidad Adventista de Montemorelos.

Meza Escobar, M. (2011). El marketing educativo, la imagen institucional y el apoyo de los padres como predictores de la actitud de demanda de la Educación Universitaria Adventista. México: Tesis de doctorado no publicada. Universidad Adventista de Montemorelos.

Miranda, D. (2010). La Imagen Exterior de España, tal como España la ve. Madrid: Tesis de doctorado no publicada. Universidad Complutense de Madrid.

Pérez, V. (1993). Cultura organizacional y valores profesionales. Tesis doctoral no publicada. Madrid: Universidad Complutense de Madrid

Perozo de Jiménez, G. (2004). Gestión comunicacional e imagen corporativa en la Universidad Nacional Abierta (UNA). Revista de Ciencias Sociales (RCS ), X(2), 295-307.

Reniham, F., Reniham, P. (1989). Institucional Image: The Concept and Implications for Administrative Action. NASSP Bulleting. (Marzo, 89).

Salazar Estrada, J. G., Guerrero Pupo, J. C., Machado Rodríguez, Y. B., \& Cañedo Andalia, R. (2009). Clima y cultura organizacional: dos componentes esenciales en la productividad laboral. ACIMED, 20(4), 67-75. Retrieved from http://scielo.sld.cu/scielo.php?script=sci_arttext\&pid=S1024$94352009001000004 \& \operatorname{lng}=\mathrm{es} \& n \mathrm{rm}=$ iso\&tlng=es

Simon, R. (1986). Relaciones Públicas: Teoría y Práctica. México: Editorial Limusa, S.A. de C.V.

Smirnov, A. (1960). Psicología. 4ta ed. México: Editorial Grijalba

Solís-Hurtado, J. (2004). El manejo del marketing en el servicio educativo; en la organización educativa continental. Lima: Tesis de pregrado no publicado. Universidad Nacional Mayor de San Marcos.

Topor, R. (1983). Marketing Higher Education. Washinton, D.C: Council for Advancement and Support of education.

Topor, Robert. (1986). Institucional Image: How to define, improve, market it. USA: Council for Advancement and Suport of Education

Turpo, J. (2011). Misión educacional de la Universidad Peruana Unión y su percepción en públicos selectos. Apunt. Univ. 1(1), 45-58.

Yasky, D. N. (2011). Los públicos de las comunicaciones financieras. Cuadernos Del Centro de Estudios En Diseño Y Comunicación. Ensayos, (35), 65-83. Retrieved from http://www.scielo.org.ar/scielo.php?script=sci_arttext\&pid=S1853$35232011000100007 \& \operatorname{lng}=\mathrm{es} \& \mathrm{nrm}=\mathrm{iso} \& \mathrm{t} \operatorname{lng}=\mathrm{es}$ 